OPEN ACCESS

Edited by:

Patricia Krawczak, IMT Lille Douai, France

Reviewed by:

Yaodong Liu,

Institute of Coal Chemistry (CAS),

China

Wei Xu,

IMDEA Nanociencia, Spain

*Correspondence:

Hamideh Mirbaha

hamideh.mirbaha@unitn.it

Nicola M. Pugno

nicola.pugno@unitn.it

Specialty section:

This article was submitted to Polymeric and Composite Materials,

a section of the journal

Frontiers in Materials

Received: 29 April 2020 Accepted: 22 June 2020

Published: 24 July 2020

Citation:

Mirbaha H, Scardi P, D'Incau M,

Arbab S, Nourpanah P and

Pugno NM (2020) Supramolecular

Structure and Mechanical Properties of Wet-Spun Polyacrylonitrile/Carbon

Nanotube Composite Fibers

Influenced by Stretching Forces.

Front. Mater. 7:226

doi: 10.3389/fmats.2020.00226

\section{Supramolecular Structure and Mechanical Properties of Wet-Spun Polyacrylonitrile/Carbon Nanotube Composite Fibers Influenced by Stretching Forces}

\author{
Hamideh Mirbaha ${ }^{1,2 *}$, Paolo Scardi ${ }^{3}$, Mirco D'Incau ${ }^{3}$, Shahram Arbab4, \\ Parviz Nourpanah ${ }^{2}$ and Nicola M. Pugno ${ }^{1,5 *}$
}

${ }^{1}$ Laboratory of Bio-Inspired, Bionic, Nano, Meta Materials \& Mechanics, Department of Civil, Environmental and Mechanical Engineering, University of Trento, Trento, Italy, ${ }^{2}$ Department of Textile Engineering, Amirkabir University of Technology, Tehran, Iran, ${ }^{3}$ Department of Civil, Environmental and Mechanical Engineering, University of Trento, Trento, Italy, ${ }^{4}$ Advanced Textile Materials and Technology Research Institute, Department of Textile Engineering, Amirkabir University of Technology, Tehran, Iran, ${ }^{5}$ School of Engineering and Materials Science, Queen Mary University of London, London, United Kingdom

The effect of different elongation conditions on the crystalline structure and physical and mechanical properties of polyacrylonitrile/carbon nanotube (PAN/CNT) microfibers during the wet spinning process was studied. It turns out that the response of polymer chains in PAN/CNT and in PAN fibers to the stretching forces from jet stretching and steam drawing is different. The $X$-ray diffraction $(X R D)$ results showed that the crystalline domain size in steam-drawn PAN/CNT fibers is 1.5 times larger than in PAN fibers. CNTs alter the optimum stretching conditions, as they improve the crystalline structure of the PAN/CNT fibers at lower steam drawing ratios than PAN fibers, through nucleation of crystals on their surface. Synchrotron-radiation XRD studies revealed that the presence of CNTs improves the crystal orientation of PAN/CNT fibers significantly. In addition, steam drawing is more effective in improving the crystal orientation than jet stretching. The mechanical properties of PAN/CNT fibers have also been affected by steam drawing more than jet stretching. Multiwalled CNTs have the biggest impact on Young's modulus. The Young's modulus of PAN/CNT fibers could increase up to 19\% higher than PAN fibers at specific stretching conditions, i.e., steam drawing ratio of 2.5. Better orientation of polymers and crystals in the fiber direction is the reason for the enhancement of Young's modulus. To our knowledge, the differences between the response of PAN/CNT and PAN fibers to stretching forces inside coagulation bath and after fiber coagulation as well as the difference in evolution of crystalline structure at different stretching stages has not been reported elsewhere.

Keywords: PAN/CNT composite fibers, wet spinning, jet stretch, steam drawing, crystalline structure, mechanical properties 


\section{INTRODUCTION}

Incorporation of CNTs in PAN microfibers has the potential to improve the physical, mechanical, electrical, and thermal properties of PAN fibers. This can be achieved without any significant weight change and or need to use different production equipment (Brown et al., 2014; Sahin et al., 2014; Li et al., 2018; Mirbaha et al., 2019). These fibers have the potential to be used as a carbon fiber precursor (Mikolajczyk et al., 2010; Sahin et al., 2014). By addition of CNTs, Li et al. (2015) obtained 35 and $17 \%$ improvement in tensile strength and tensile modulus of PAN fibers, respectively. To have PAN/CNT fibers with better mechanical properties, the internal structure of fibers needs to be controlled and designed (Ji et al., 2007). The formation of a fiber structure in the wet spinning process is a very important step in determining the properties of produced fibers (Wilms et al., 2013; Wei et al., 2020). Since there are many effective parameters on the evolution of a fiber structure in the wet spinning stage, the favored production of PAN/CNT composite fibers with promising properties involves thorough research on the spinning process, which is also the main aim of this work.

PAN/CNT composite fibers can be produced by the wet spinning method (Mirbaha et al., 2019). During the wet spinning process, shear and elongational forces are applied on filaments, affecting the alignment of polymer chains (Mai et al., 2011; Dintcheva et al., 2011). Shear stress is applied on the spinning dope while it flows inside the spinneret (Mikolajczyk et al., 2010). According to Cao et al. (2004), a strong enough shear force can align polymer chains in the flow direction. The melt spinning process of polypropylene/CNT composite fibers was studied by Kang and Ryu (2013). They observed that shear stress applied to the spinning dope can also be transferred to CNTs, resulting in their orientation in the flow direction. Minus et al. (2012) observed that CNTs can act as nucleating agents for the crystallization of polyethylene. A small amount of CNT dispersed within a polymer can influence its orientation and crystallinity.

Stretching is one of the most necessary steps in fiber production, leading to improvement in molecular orientation along the fiber direction as well as fineness, tensile strength, and modulus (Zeng et al., 2007; Arbab et al., 2011a). The study on the effect of elongational forces on the initial structure of the PAN fibers in the presence of CNTs can help control the alignment of polymers and CNTs, and the formation of an interphase between them, subsequently playing an important role on the final properties of the fiber (Lin et al., 2019). Fiber stretching can be done at different production stages including jet stretching inside the coagulation bath and hot drawing of coagulated fibers in different environments (Arbab et al., 2011a; Lian et al., 2012). Jet stretching reduces the residence time of filaments in the coagulation bath, decreasing the available time for formation and growth of voids (Arbab et al., 2011b). Arbab et al. (2011b) obtained a denser structure with smaller voids, when they increased the jet stretch inside the coagulation bath of the wet spinning process of PAN fibers. Li et al. (2015) observed that drawing has two opposite effects on the crystallization of PAN fibers. Applying stretch results in orientation assisted crystallization and crystallinity improvement. On the other hand, increasing the stretch ratio shortens the crystallization step and reduces the crystallinity. According to Cao et al. (2004), the stretching force inside the coagulation bath is able to unwind the random coils inside fiber filaments, stretch the chains, improve the chain orientation, and increase the compactness of the structure. While shear forces inside the spinneret can cause the alignment and orientation of polymer chains, the coexistence of shear and elongational forces results in stretching and orientation of polymer chains and opening of random coils (Cao et al., 2004). According to Mai et al. (2011), polymer chains and CNTs are likely to change from an entangled shape to a stretched form under elongational force. Polymer chains and CNTs have a greater chance of interaction in their stretched form. Polymer chains can crystallize around CNTs, thus producing a better interface interaction. Mai et al. (2011) reported better dispersion of CNTs at higher stretch ratios during melt spinning of PA66 as well as better orientation of fibers in the presence of CNTs.

After formation of a fiber structure in the coagulation bath, it is possible to improve the orientation and fiber properties by hot drawing the as-spun fibers (Zeng et al., 2007). The time scale of the reaction of polymer chains to hot drawing of coagulated fibers is different from the jet stretching of the polymer fluid (Zeng et al., 2007). During the hot drawing stage, the orientation of a fibrillar network and polymer chains in amorphous and crystalline regions increases, improving the mechanical strength (Arbab et al., 2011b).

Most of the researches in the field of PAN/CNT composite fiber production have focused on the structure and properties of the final products, while studies on the evolution of the structure during the production phases are still limited. The coexistence of shear and elongation in the wet spinning stage alters the orientation and placement of the polymer chains and CNTs and determines the initial fiber structure that can be developed during the next production stages. In a previous work, we reported the different effects of shear rates and jet stretching ratios inside the coagulation bath - where the filaments are still not fully solidified - on the morphology, crystalline structure, and physical and mechanical properties of as-spun PAN/CNT fibers (Mirbaha et al., 2019). In this work, we kept the shear rate constant, while applying a new stretching step in water vapor after full fiber coagulation. The objective of this research is the exploration of the effect of elongational forces at different stretching conditions on the formation and evolution of the PAN/CNT fiber structure and properties affected by the presence of CNTs. Understanding the effect of the mentioned variables can be regarded as a scheme part to control the spinning process and improve the physical and mechanical properties of the final PAN/CNT fibers. For this reason, the effect of jet stretching and hot drawing on the structure and properties of PAN/CNT composite fibers is studied using physical and mechanical measurements, X-ray diffraction, and FESEM microscopy. The impact of the existence of CNTs inside fibers on structural development is also explored in comparison with PAN reference fibers. 


\section{EXPERIMENTAL}

\section{Materials}

PAN powder with methyl acrylate and sodium methallyl sulfonate comonomers was obtained from Polyacryl Corp., Iran. The viscosity molecular weight was $112,000 \mathrm{~g} / \mathrm{mole}$. Analysisgrade dimethyl formamide (DMF) solvent was obtained from Merck Co., Germany, and was used as received. Multiwalled CNTs were obtained from the Research Institute of Petroleum Industry, Iran. The average length and diameter of nanotubes are 10 micron and 10-30 nanometers, respectively. The purity was over $95 \%$.

\section{Methods \\ Preparation of the Spinning Dope}

The spinning dope for reference PAN fibers was prepared from dissolving PAN powder in DMF solvent at a concentration of $20 \% \mathrm{w} / \mathrm{v}$. The spinning dope for PAN/CNT composite fibers was prepared by dissolving PAN powder in DMF solvent at a concentration of $20 \% \mathrm{w} / \mathrm{v}$, as in the spinning dope for PAN fibers. Based upon the literature review and optimization tests, the CNT concentration of $0.75 \%$ wt. proportional to the weight of the PAN polymer was chosen. For production of $35 \mathrm{cc}$ of polymer solution, $0.0525 \mathrm{~g}$ CNT was added to $400 \mathrm{~mL}$ of DMF solvent. CNTs were dispersed for $30 \mathrm{~min}$ in DMF solvent using a UP200S ultrasonic homogenizer (Hielscher Ultrasonics $\mathrm{GmbH}$, Germany). Then, a mechanical stirrer was placed in the CNT/DMF dispersion and polymer solution was continuously added to the dispersion under shear force until a homogenous solution was attained. The composite solution was further homogenized for $30 \mathrm{~min}$ using a ultrasonic homogenizer. An optical image of the final state of composite solution after ultrasonication is shown in Figure 1. Using mild heating and shear force, the extra solvent was evaporated and the final $\mathrm{PAN} / \mathrm{CNT} / \mathrm{DMF}$ solution with PAN and CNT concentrations of 20 and $0.75 \%$, respectively, was attained. For determination of the end point of solvent evaporation, a finely scaled narrow glass container was used and the volume reduction during evaporation was closely observed until the final volume of the spinning solution was achieved.

\section{Wet Spinning}

A laboratory wet spinning machine was used to produce composite PAN/CNT and reference PAN fibers. A schematic diagram of this wet spinning machine is reported in our previous paper (Mirbaha et al., 2019). A metering pump was used to pump the spinning dope at the constant shear rate of $8,000 \mathrm{~s}^{-1}$ to the coagulation bath through a one-hole spinneret with a diameter of 200 microns. The coagulation bath contained water as nonsolvent and the temperature was kept at $20^{\circ} \mathrm{C}$ to reduce the phase separation speed. The freely spun fibers were produced without any stretching force applied on the filaments. These fibers were collected from the coagulation bath and then were thoroughly washed and dried at room temperature. Jet-stretched fibers were picked up at take-up rollers. The take-up velocity to the extrusion velocity of the spinning dope is defined as the jet stretch ratio. Jet stretch ratios of 2 and 2.5 were applied

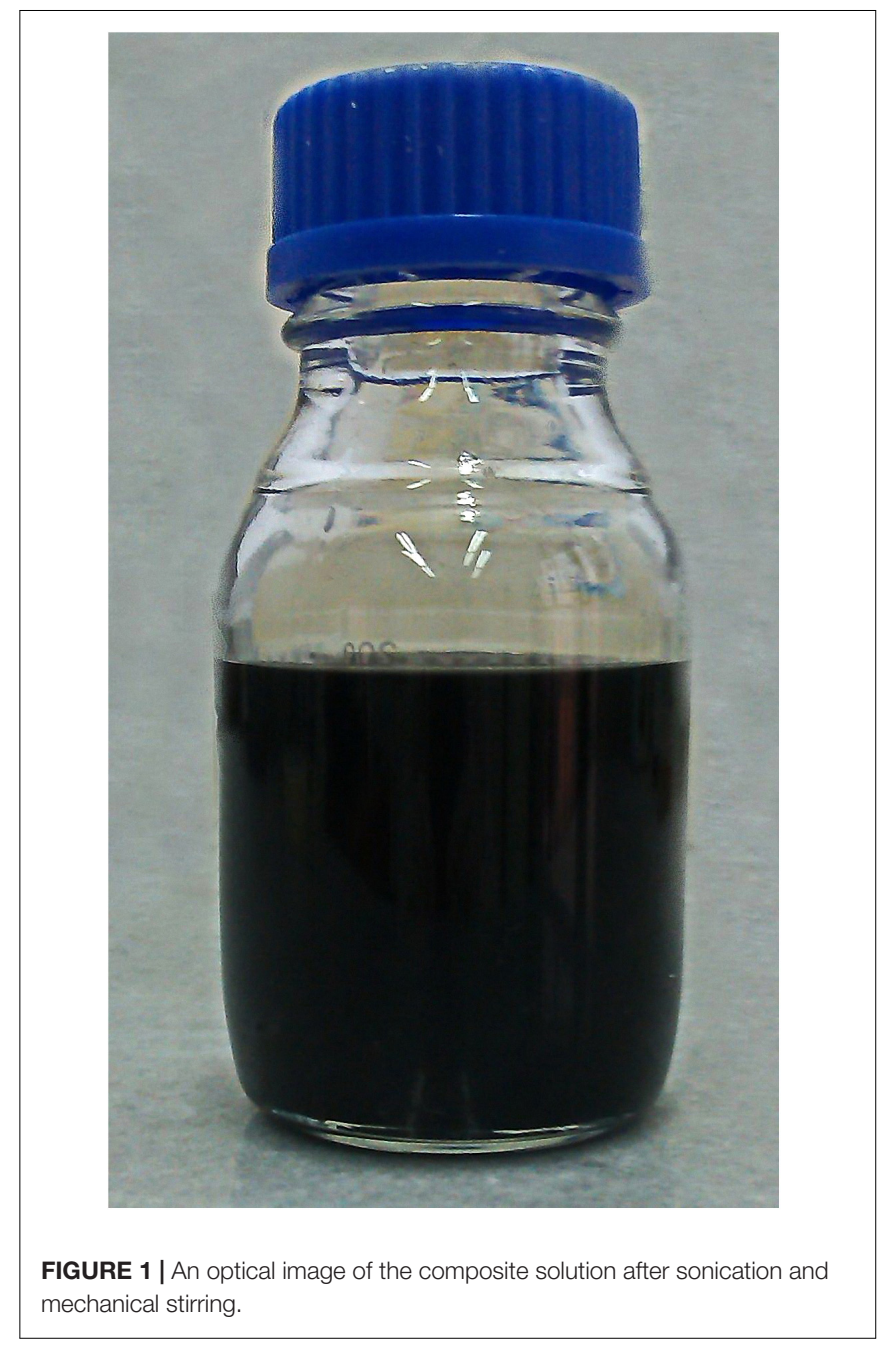

on fibers by changing the take-up speed in relation to the extrusion velocity. Jet-stretched fibers were washed thoroughly with water, and they were dried naturally in air. The hot drawing stage was performed in water vapor at $100^{\circ} \mathrm{C}$ in a laboratory-designed drawing machine (Figure 2). Fibers were fixed between two steel grips inside the hot steam chamber and were drawn up to different drawing ratios with constant speed of $1.3 \mathrm{~mm} / \mathrm{sec}$. The drawn fibers were then dried at room temperature.

The sample names and production conditions of PAN/CNT and PAN fibers are listed in Table 1. The letters of P and PC in sample names represent PAN and PAN/CNT, respectively. The numbers coming after the letters of $\mathrm{S}$ and $\mathrm{D}$ are jet-stretch ratios inside the coagulation bath and steam-draw ratios, respectively. The fiber codes without these numbers are freely spun fibers. Steam-draw ratios are defined as the final length of fibers after drawing in hot water vapor at $100^{\circ} \mathrm{C}$ to the initial length of fibers before drawing. The freely spun and jet-stretched fibers are similar to those reported in our previous work (Mirbaha et al., 2019). They are reported in this work for comparison to steam-drawn fibers. 


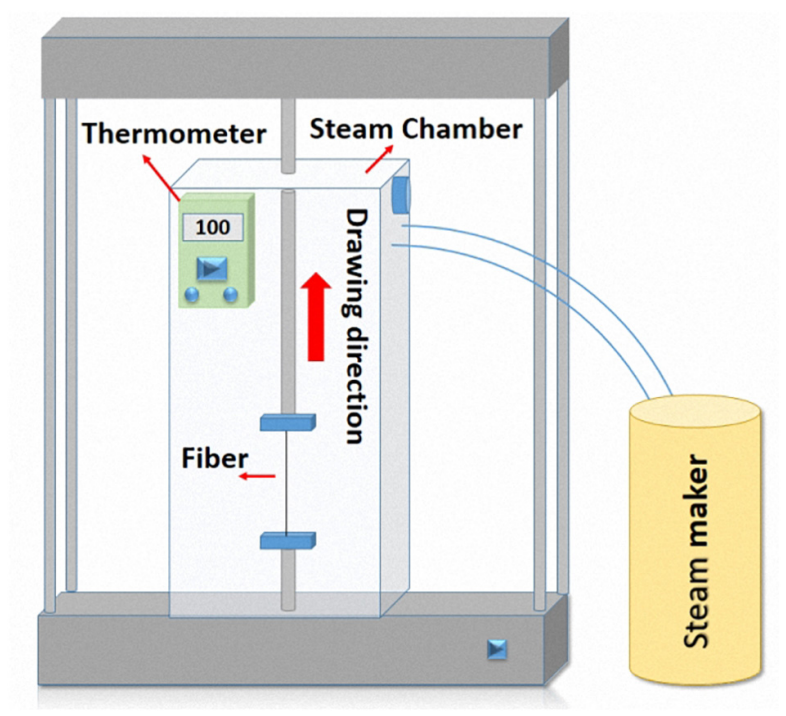

FIGURE 2 | Schematic diagram of the steam drawing chamber used in this research.

TABLE 1 | Denotation and production conditions of PAN/CNT and PAN fibers.

\begin{tabular}{lcccc}
\hline Condition & \multicolumn{2}{c}{ Sample names } & Jet stretch ratio & Jet stretch ratio \\
\cline { 2 - 3 } & PAN/CNT & PAN & & \\
\hline Freely spun & PC & P & - & - \\
Jet stretched & PC-S2 & P-S2 & 2 & - \\
& PC-S2.5 & P-S2.5 & 2.5 & - \\
Steam srawn & PC-D2.5 & P-D2.5 & - & 2.5 \\
& PC-D5 & P-D5 & - & 5 \\
& PC-S2-D5 & P-S2-D5 & 2 & 5
\end{tabular}

\section{Characterization}

In order to evaluate the dispersion of CNTs and determine the optimum dispersion conditions, UV-visible analysis was utilized. For this purpose, the PAN/DMF solution with a concentration of $20 \%$ and the CNT/DMF suspension with a CNT concentration of $0.75 \%$ proportional to the weight of the PAN polymer were prepared. Similar to preparation of the spinning dope, the CNT/DMF solution was first ultrasonicated for $30 \mathrm{~min}$. Then, by the help of a mechanical stirrer, the polymer solution was added to the CNT/DMF dispersion under shear force. The dispersion process was again continued for 15, 30, 60, and $75 \mathrm{~min}$. At each of these times, a sample was collected, diluted, and tested in a Cary 100 UV-visible spectrophotometer (Agilent, United States) in a wave number ranging from 200 to $700 \mathrm{~nm}$.

A Microphot FXA optical microscope (Nikon, Japan) at a magnification of $50 \times$ was used to measure the fiber diameter at several points along the fiber. The average values of different replicates are reported here. A GR-200 balance (A and D Balance, Japan) was used to measure the mass of different fibers at various lengths, and the average value of linear density was calculated and reported in unit of tex as the mass in grams of 1,000 meters of fibers. The overall porosity of fibers was approximately calculated as follows (Arbab et al., 2008):

$$
\text { Overall porosity }(\%)=\frac{\pi R^{2} L-m \rho^{-1}}{\pi R^{2} L} \times 100
$$

where $R, L$, and $m$ are the radius, length, and mass of fibers, respectively, and $\rho$ is the polymer density.

The microscopic images of fiber cross sections at magnifications of $500 \times, 7,000 \times$, and $35,000 \times$ were obtained using a MIRA3 FESEM (Tescan, Czech Republic). The operation voltage was $15 \mathrm{kV}$. The samples were coated by gold, and the intact cross sections were cut in liquid nitrogen.

A Panalytical X'Pert MRD X-ray diffractometer (United Kingdom) was utilized to study the crystalline parameters of PAN/CNT and PAN fibers. The diffractometer worked at an operation voltage of $40 \mathrm{kV}$ and current of $40 \mathrm{~mA}$. Co $\mathrm{K} \alpha$ radiation at wavelength of $1.790 \AA$ was used. Fibers were placed as bundles on the sample holder. The scanning range was $10^{\circ}-48^{\circ}(2 \theta)$, the scanning step was $0.15^{\circ}$, and the counting time was $25 \mathrm{~s} / \mathrm{step}$. To extract data from X-ray patterns, the diffraction peaks were resolved using Origin 2017 software (OriginLab, United States) based on three Lorentzian functions. The crystalline parameters were calculated from the intense peak at $2 \theta \sim 17^{\circ}$. Bragg law was used to calculate the plane spacing (d), and lateral crystallite size $\left(L_{c}\right)$ was estimated using the Scherrer equation (Ji et al., 2007; Gong et al., 2014):

$$
L_{C}=\frac{K \lambda}{\beta \cos \theta}
$$

where $K$ is a constant with a value of 0.89 and $\beta$ is the full width at half maximum (FWHM) intensity of the peak.

The degree of crystallinity was assessed using Gupta-Singhal's method (Ouyang et al., 2015):

$$
\text { Crystallinity }=\frac{A_{\text {crystal }}}{A_{\text {total }}} \times 100
$$

where $A_{\text {crystal }}$ is the area of crystalline peaks and $A_{\text {total }}$ is the total area of diffraction after subtracting the background.

Synchrotron X-ray patterns were collected at the MCX beamline of Elettra synchrotron in Trieste, Italy (Rebuffi et al., 2014). A beamline energy of $17.5 \mathrm{keV}$ was used. The single fiber was placed horizontally with its axis perpendicular to X-ray radiation beam. Therefore, the vertical and horizontal directions in two-dimensional (2D) patterns are related to equatorial and meridional directions of fibers, respectively. 2D X-ray diffraction patterns were integrated to two one-dimensional (1D) equatorial and meridional scans. The intensity and area under the peak were calculated by modeling with a Python script. The modeling is made using a decaying exponential background and a pseudoVoigt peak profile.

A universal testing machine MIDI 10 (Messphysik Materials Testing $\mathrm{GmbH}$, Austria) was used to measure the mechanical properties of single threads of PAN and PAN/CNT fibers. A 10-N load cell was utilized. The initial distance between two steel grips was $1 \mathrm{~cm}$. An elongation rate of $0.1 \mathrm{~mm} / \mathrm{s}$ based on Standard D3822 was chosen. The mechanical properties reported in this paper are the average values of at least 10 test replicates. The 
coefficient of variation (\% CV) was calculated for each set of tests. The breaking tenacity was measured from the load at break divided by the linear density of fibers in tex. Young's modulus was measured from the slope of the initial linear section of a specific stress-strain curve. The mechanical properties are reported in $\mathrm{cN} /$ tex (force divided by mass per unit length) instead of $\mathrm{MPa}$, as the cross section of some fibers cannot be easily defined. Considering 1 tex $=0.001 \mathrm{~g} / \mathrm{m}$, tensile data in $\mathrm{cN} /$ tex (force per unit linear density) can be converted to $\mathrm{Pa}$ (force per unit area of cross section) by multiplying by the density of the fiber in proper units (Ward, 1997).

\section{RESULTS AND DISCUSSION}

\section{Determination of Optimum Dispersion Time}

CNTs tend to form aggregates in solution due to Van der Waals forces. Therefore, one of the challenges for producing PAN/CNT composite fibers is dispersion of CNTs in the form of individual tubes or in small bundles inside the viscous polymer matrix (Basu-Dutt et al., 2012). At these conditions, the interface area between CNT and polymer increases, leading to improvement in fiber mechanical properties.

The addition of polymer to dispersion solution after $30 \mathrm{~min}$ of sonication is due to the fact that polymer chains can be adsorbed on the surface of CNTs and penetrate to their bundles with the help of ultrasonic force (Bakhtiary Davijani and Kumar, 2015). Therefore, CNT bundles can become smaller. As the dispersion time increases, the PAN/CNT/DMF dilute solution becomes darker, while the aggregations that can be recognized with the naked eye decrease. These aggregations completely disappear at dispersion times of 60 min or longer (Figure 3A).

UV-visible spectra of PAN/CNT/DMF solutions contain one absorption peak at a wavelength of about $265 \mathrm{~nm}$ (Figure 3B). Since only individual CNTs can absorb UV and visible lights, the maximum absorbance during dispersion can be used as a method to evaluate the dispersion state of CNTs (Sapalidis et al., 2018). The maximum UV-visible absorbance increases with an increase in dispersion time (Figure 3B). At dispersion time of $60 \mathrm{~min}$ or longer, the maximum absorbance stays constant, revealing that maximum possible dispersion of CNTs is achieved. Increasing the dispersion time to longer than 60 min not only does not improve the dispersion state of CNTs in the solution but also increases the energy consumption as well as the possibility of undesired damages to the CNTs. As a result, dispersion period of $60 \mathrm{~min}$ was used in this research.

\section{Physical Properties}

The existence of CNTs among PAN polymer chains causes a decrease in the fiber diameter in all spinning and stretching conditions, i.e., freely spun, jet-stretched, and steam-drawn fibers (Table 2). The diameter decrease is attributed to the smaller die swell as a result of the effect of CNTs on lowering the elastic recovery of polymer chains (Mirbaha et al., 2019). CNTs decrease the elastic recovery of polymer chains by reducing the chain mobility and formation of the interphase between polymers and CNTs. Consequently, polymer chains can preserve their orientation better in the presence of CNTs.

At identical stretch ratios, the reduction in diameter and linear density after steam drawing is less than that of jet stretch inside the coagulation bath (Table 2). For example, the diameter of steam-drawn PAN/CNT fibers at the ratio of 2.5 is about 1.5 times larger than that of jet-stretched PAN/CNT fibers at the ratio of 2.5 .

The porosity of PAN fibers increases when CNT exists in the structure. The high porosity of fiber samples shows that the spinning conditions need further optimization. The overall fiber porosity is attributed to macrovoids (finger-like voids between fibrillar parts) as well as nanovoids (among fibrils) (Arbab et al., 2008). The more voids are present in the structure, the more random and disordered will be the arrangement of molecular chains, leading to less fiber orientation (Ji et al., 2007). The biggest difference between porosity of PAN/CNT and PAN fibers is observed when fibers are jet stretched (Table 2). For PAN reference fibers, jet stretch has brought structural elements close to each other reducing the voids, whereas for PAN/CNT fibers the void volume has increased by application of jet stretch. In addition, the presence of CNTs among polymer chains and formation of interphase with polymers has probably prevented from free movement of chains, intensifying the formation of voids under stretching forces inside coagulation bath. The higher porosity of PAN/CNT fibers compared to PAN fibers could also possibly be due to increased solution elasticity. The smallest difference between porosity of PAN/CNT and PAN fibers is observed when fibers are spun under steam drawing. Steam drawing has increased the porosity of PAN fibers sharply compared to jet-stretched fibers, whereas the difference between porosity of jet-stretched and steam-drawn PAN/CNT fibers is negligible. These results show the different response of PAN/CNT and PAN fibers to jet stretch and steam drawing. The lowest porosity of PAN/CNT fibers is obtained when fibers are jet stretched at the ratio of 2 and then steam drawn at the ratio of 5 . The combination of jet stretch and steam drawing results in diameter reduction, formation of smaller voids during coagulation, and narrowing and healing of the voids in water steam, leading to a more compact structure.

The porosity of P-S2-D5 has increased compared to P-S2 fibers, whereas the opposite trend is observed in PC fibers. Jet stretching has brought the structural parameters in PAN fibers close to each other, reducing the voids and porosity of structure. However, jet stretching at high ratios used in this research, which turned out to be higher than optimum values for PAN/CNT fibers, has increased the porosity of PAN/CNT fiber structure. Steam drawing at temperatures higher than glass transition temperatures has been more successful in healing the pores and voids in the structure of PAN/CNT fibers. This may be due to lower crystallinity of the structure of jet-stretched PAN/CNT fibers (as it will be discussed in section "Laboratory X-Ray Diffraction"), which makes the structural arrangement and orientation easier than PAN fibers. In PAN fibers, however, steam drawing at the temperature and rate used in this research has not reduced the porosity of structure. This might result from chain breakage during steam drawing and/or slippage of 

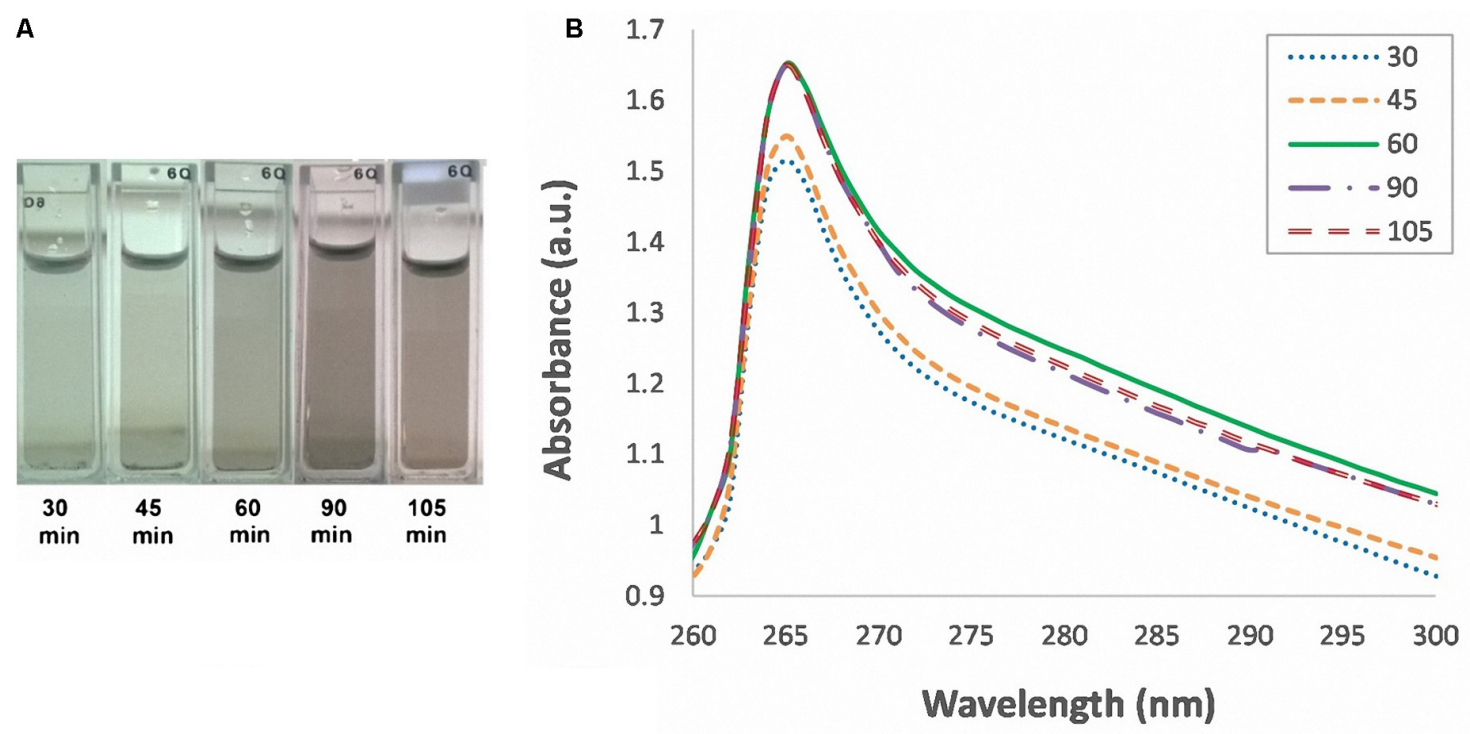

FIGURE 3 | (A) Dilute PAN/CNT/DMF solutions after ultrasonication at different times. (B) UV-visible spectrum of PAN/CNT/DMF solutions at different ultrasonication times.

some fibrils and crystalline domains to rearrange and reorganize new domains, introducing new voids in the structure. Liu et al. (2015) also observed an increase in porosity at some hot drawing ratios and lower drawing temperatures during the production of ultra-high molecular weight polyethylene fibers.

\section{Fiber Morphology}

During the coagulation process of PAN fibers, a structure consisting of loose fibrils and finger-like voids along the radius of the fiber is formed. This structure is formed because of counter diffusion of solvent/non-solvent and rapid phase separation (Ouyang et al., 2011). Finger-like voids reduce the mechanical properties of the fibers (Arbab et al., 2008). Application of jetstretch ratios of 2 and 2.5 to PAN/CNT fibers makes the cross

TABLE 2 | Physical properties of PAN/CNT and PAN fibers at different spinning conditions.

\begin{tabular}{lcccccc}
\hline Condition & $\begin{array}{c}\text { Sample } \\
\text { name }\end{array}$ & $\begin{array}{c}\text { Diameter } \\
(\boldsymbol{\mu} \mathbf{m})\end{array}$ & $\begin{array}{c}\text { Linear } \\
\text { density (tex) }\end{array}$ & $\begin{array}{c}\% \text { CV } \\
\text { Porosity } \\
(\%)\end{array}$ \\
\hline PAN/CNT & & & & & & \\
Freely spun & PC & 275.0 & 0.04 & 15.1 & 0.05 & 78.5 \\
Jet stretched & PC-S2 & 147.9 & 0.07 & 4.0 & 0.09 & 80.3 \\
& PC-S2.5 & 138 & 0.13 & 2.8 & 0.16 & 84.1 \\
Steam Drawn & PC-D2.5 & 197.6 & 0.04 & 6.3 & 0.07 & 82.6 \\
& PC-D5 & 157 & 0.06 & 3.3 & 0.08 & 85.5 \\
& PC-S2-D5 & 77.4 & 0.15 & 1.1 & 0.19 & 79.1 \\
PAN & & & & & & \\
Freely spun & P & 335.4 & 0.05 & 28.2 & 0.06 & 73.1 \\
Jet stretched & P-S2 & 185.5 & 0.06 & 10.0 & 0.05 & 68.7 \\
& P-S2.5 & 164.2 & 0.11 & 7.3 & 0.05 & 70.7 \\
Steam Drawn & P-D2.5 & 241.4 & 0.05 & 12.4 & 0.06 & 77.2 \\
& P-D5 & 196.2 & 0.05 & 6.8 & 0.07 & 81.1 \\
& P-S2-D5 & 102 & 0.04 & 2.1 & 0.06 & 76.1
\end{tabular}

section irregular with deep surface grooves (Figure 4 in Mirbaha et al., 2019). On the other hand, application of steam drawing with the ratio of 5 to jet-stretched PAN/CNT fibers heals the surface grooves to a large extent, Figure 4A. The cross section of these fibers is also more regular compared to jet-stretched PAN/CNT fibers (Mirbaha et al., 2019). The discrepancy of the shape of the cross section from a circular form affects the mechanical properties of the fibers (Chen et al., 2010). PAN reference fibers at jet stretch ratios of 2 and 2.5 have a circular cross section (Figure 4 in Mirbaha et al., 2019). Application of a steam drawing ratio of 5 to these fibers also does not change the cross section shape significantly (Figure 4B). This difference suggests that the tolerability of PAN/CNT fibers to jet stretch is less than that of PAN fibers. The voids in the cross section of steam-drawn fibers are shrunk as a result of elongational forces. In addition, the fibrils are more oriented and compact after steam drawing. Fibrillar compactness of PAN/CNT fibers is more than that of PAN fibers. This is attributed to the smaller die swell in PAN/CNT fibers (Mirbaha et al., 2019). As it was reported in our previous paper (Mirbaha et al., 2019), we could not identify aggregations of CNTs in fiber cross sections even at magnifications of $150,000 \times$. This can possibly show the good state of dispersion of CNT in the matrix and wrapping individual CNTs with polymer chains (Mirbaha et al., 2019).

\section{Crystalline Structure Laboratory X-Ray Diffraction}

A sharp peak at $2 \theta \sim 17^{\circ}$ exists in the $\mathrm{X}$-ray pattern of PAN/CNT fibers, which is attributed to the diffraction peak from the (100) planes (Ouyang et al., 2015). A broad diffuse scattering appearing around $2 \theta \sim 20-30^{\circ}$ is attributed to the contribution of amorphous regions of fibers (Figure 5). These peaks in freely spun fibers are wide and weak. Application 

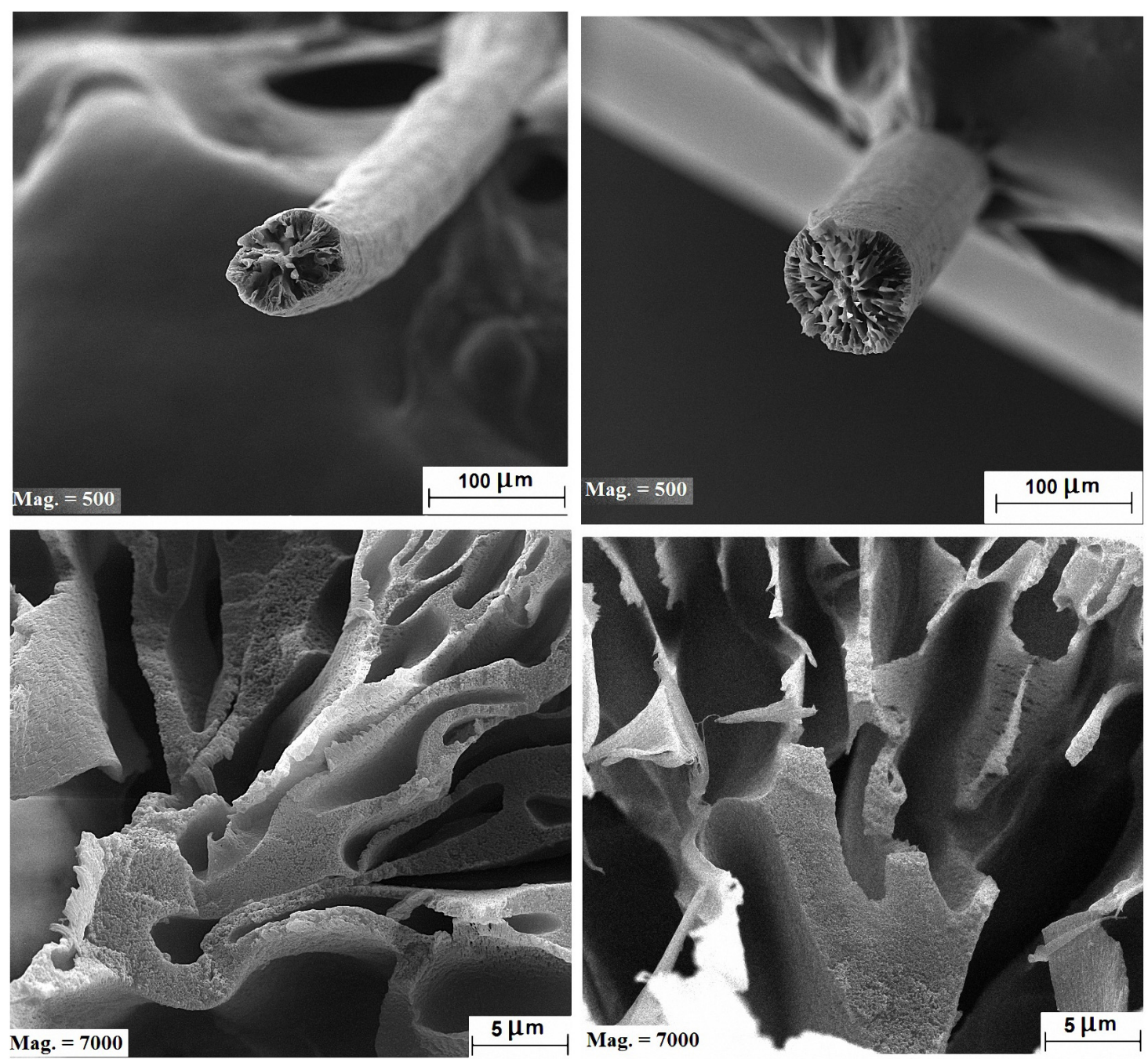

Mag. $=7000$

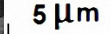

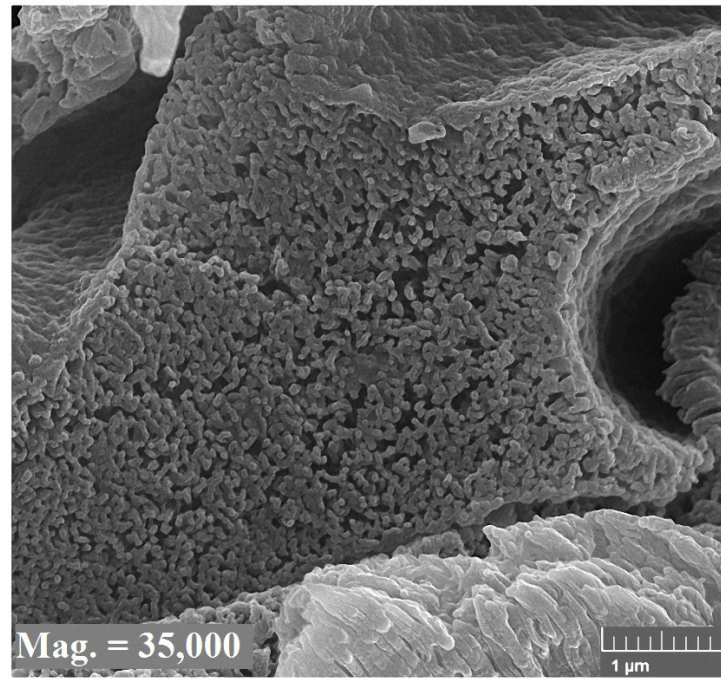

A

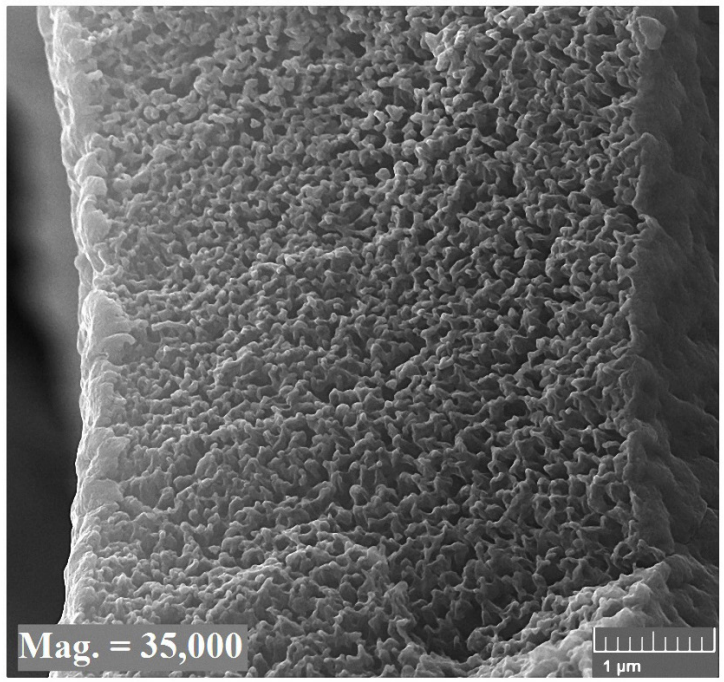

B

FIGURE 4 | FESEM images of the cross section and morphology of PAN/CNT and PAN fibers at magnifications of 500 $\times$, 7,000 $\times$, and 35,000 $\times$. (A) PC-S2-D5, and (B) P-S2-D5 
of jet stretch increases the peak intensity to some extent, whereas steam drawing sharpens the diffraction peak at $2 \theta \sim 17^{\circ}$ and increases its intensity significantly. In addition, a small crystalline peak appears at $2 \theta \sim 30^{\circ}$, showing an improvement in the crystalline structure of PAN/CNT fibers with steam drawing.

During steam drawing, polymer chains are oriented and packed in a way that orientation-induced crystallization can take place. As a result, the crystallinity and crystallite size increase sharply (Table 3). Application of steam drawing at the ratio of 2.5 has better efficiency in improving the crystalline structure of the freely spun PAN/CNT fibers compared to a total draw ratio of 10 (Table 3 ), whereas the improvement in the crystalline structure of PAN reference fibers at a steam drawing ratio of 2.5 is negligible. For improving the crystalline structure of PAN fibers and increasing their crystallite size in the absence of CNTs, application of both jet stretch and steam drawing at higher drawing ratios is necessary. To our

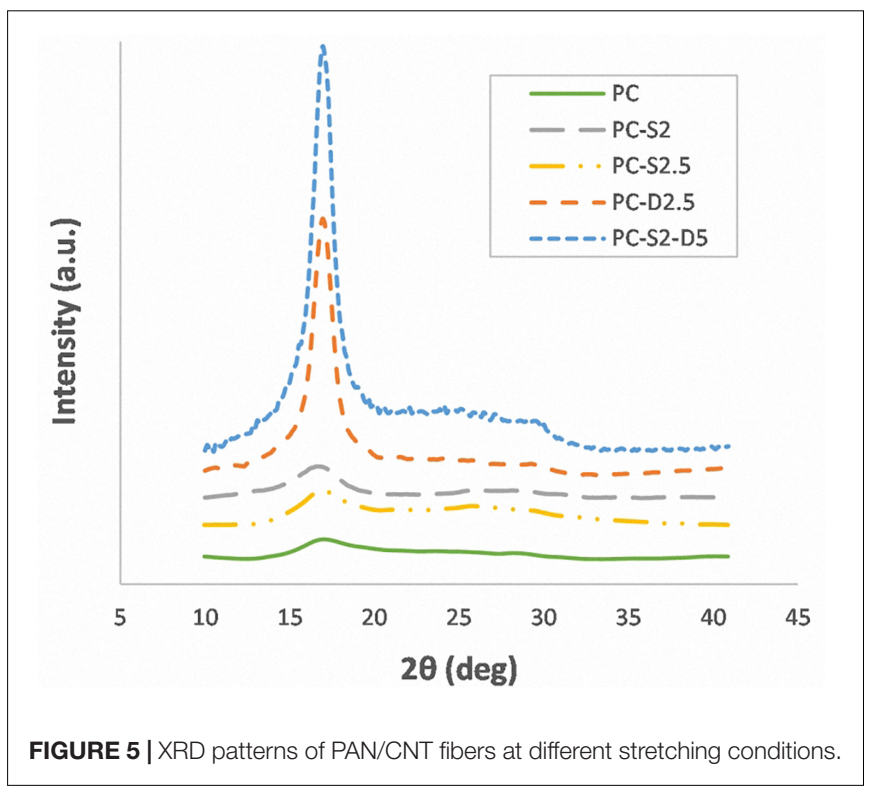

knowledge, the difference between the response of polymer chains in the composite PAN/CNT and reference PAN fibers to the stretching force inside the coagulation bath and after fiber coagulation as well as the difference in evolution of the crystalline structure at different stretching steps has not been reported elsewhere. This information can help optimize the stretching stages of PAN/CNT fibers to obtain better fiber properties.

Comparison of crystalline parameters of PC-S2.5 and PC-D2.5 shows the larger influence of steam drawing on the crystalline structure of PAN/CNT fibers compared to jet stretching (Table 3). Steam drawing has resulted in the growth of crystals and significant improvement in fiber crystallinity. As reported by Gissinger et al. (2018), this can improve the interfacial shear strength of CNTs in the matrix and result in stronger cohesion.

In the presence of the water vapor molecules at the temperature of $100^{\circ} \mathrm{C}$, polymer chains have more freedom for mobility and alignment. In addition, there is more intense nucleation and growth of crystals in the presence of CNTs, whereas PAN reference fibers show a different behavior.

Crystallite size and average crystallite layers of PAN/CNT fibers are larger than those of corresponding PAN fibers at most spinning conditions (Figure 6A). The presence of CNTs among the polymer chains facilitates the growth of crystallites by joining smaller crystallites together and joining more chains from the amorphous and transition phases to the crystal phase. We propose that nucleation and growth of PAN crystals on the surface of CNTs has resulted in improvement in the crystalline domain size. Other researchers also reported the growth of crystals in the vicinity of CNTs (Mai et al., 2011; Zhang et al., 2013).

Despite larger crystallites, the crystallinity of PAN/CNT fibers is less than that of PAN reference fibers at most spinning conditions (Figure 6B). It shows the presence of larger crystals at fewer numbers in PAN/CNT composite fibers. Jet-stretched PAN/CNT fibers have significantly lower crystallinity than corresponding PAN fibers, whereas the crystallinity of PAN/CNT fibers increases sharply with steam drawing, bringing it close to or even higher than that of PAN fibers. This shows the larger

TABLE 3 | Parameters of crystalline structure of PAN/CNT and PAN fibers at different stretching conditions.

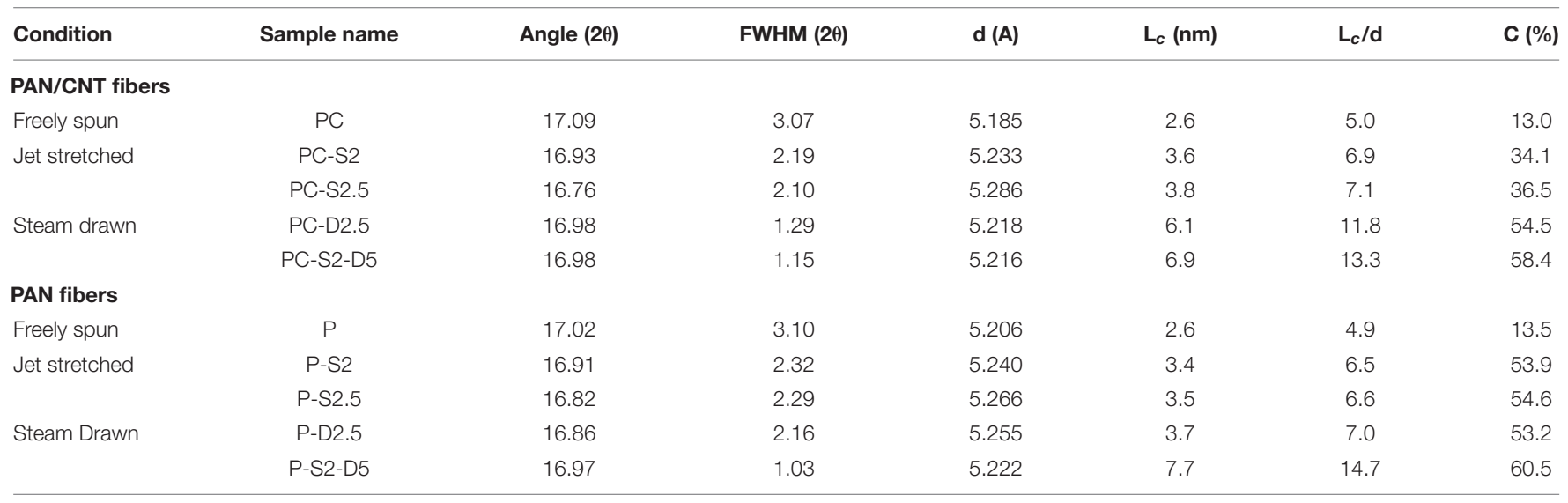

FWHM, Full width at half maximum; $d$, interplanar spacing; $L_{c}$, crystalline domain size; $L_{c} / d$, average crystallite layers; $C$, crystallinity. 


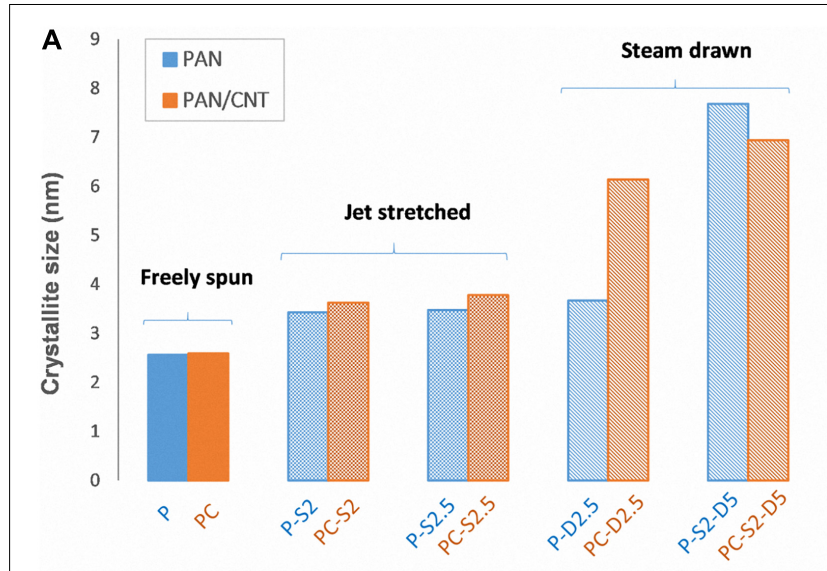

B

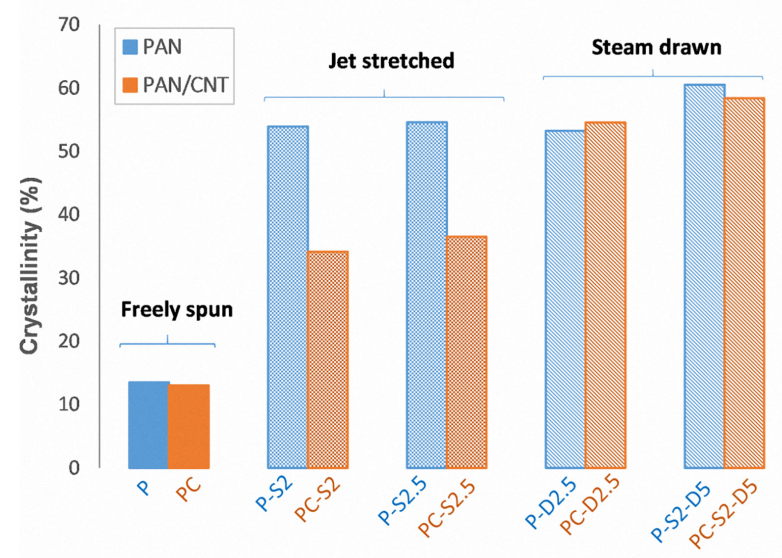

FIGURE 6 | Comparison of crystalline parameters of PAN/CNT and PAN fibers at different st retching conditions: (A) Crystallite size, (B) Crystallinity.

influence of steam drawing on crystalline evolution of PAN/CNT fibers compared to PAN fibers.

\section{Synchrotron X-Ray Diffraction}

Synchrotron radiation 2D X-ray diffraction patterns of PAN/CNT fibers and their 1D equatorial and meridional cross section profiles are shown in Figure 7. Freely spun fibers exhibited broad reflections with similar equatorial and meridional profiles, resulting from low crystallinity and irregular placement of crystals inside fibers. Application of jet stretch sharpens the peak slightly with small differences in diffraction area in equatorial and meridional directions. After steam drawing, the reflections become sharper and their intensity on the equator increases due to increased crystal perfection. Since single fibers were used for synchrotron X-ray diffraction, the intensity of diffraction ring decreases with increasing the stretching ratio and diameter reduction.

The difference between intensity $(I)$ and area $(A)$ under the peak in equatorial (eq.) and meridional (mer.) profiles can be used as a measure of crystal orientation along the fiber direction (Table 4). $I_{\text {eq. }} / I_{\text {mer }}$, and $A_{\text {eq. }} / A_{\text {mer }}$. ratios of jet-stretched PAN/CNT fibers have been increased slightly compared to freely
A
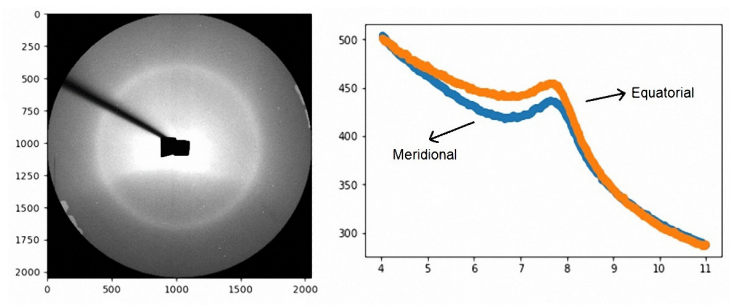

B
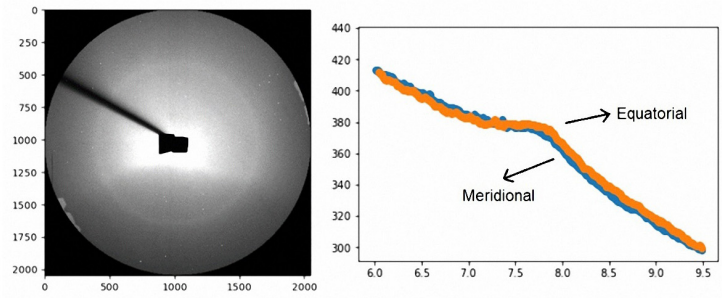

C
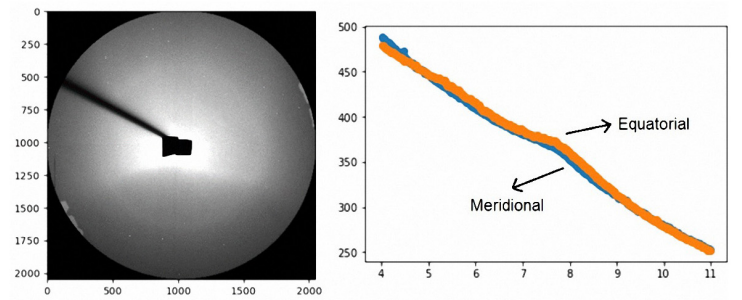

D
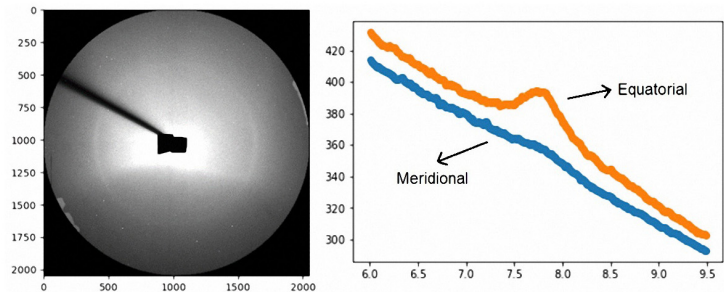

E
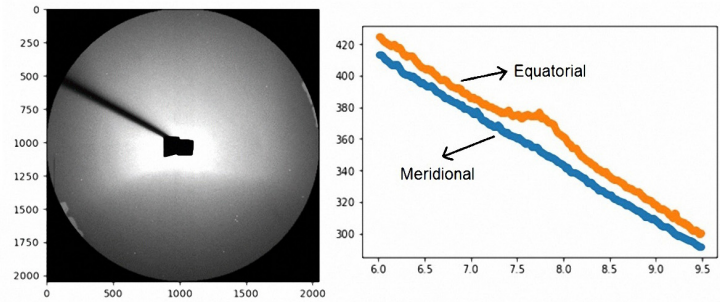

FIGURE 7 | Synchrotron radiation 2D X-ray diffraction patterns of PAN/CNT fibers at different stretching conditions and their $1 \mathrm{D}$ equatorial and meridional cross section profiles. (A) PC, (B) PC-S2, (C) PC-S2.5, (D) PC-D5, (E) PC-S2-D5.

spun fibers. Application of steam drawing with the ratio of 5 to the jet-stretched fibers increases these ratios significantly, revealing the remarkable improvement in the crystal orientation.

When PAN/CNT fibers are stretched, CNTs can also orient along the fiber direction (Dintcheva et al., 2011; Mai et al., 2011). Since crystals can nucleate in the vicinity of CNTs, increasing the orientation of CNTs improves the crystal 
TABLE 4 | Parameters extracted from equatorial ad meridional scans of synchrotron radiation X-ray diffraction patterns of PAN/CNT and PAN fibers spun at different stretching conditions.

\begin{tabular}{llcc}
\hline Sample name & Spinning condition & $\boldsymbol{I}_{\text {eq./ }} \boldsymbol{I}_{\text {mer. }}$ & $\boldsymbol{A}_{\text {eq./ }} \boldsymbol{A}_{\text {mer. }}$ \\
\hline PC & Freely spun & 1.4 & 1.49 \\
PC-S2 & Jet stretched & 1.51 & 1.58 \\
PC-S2.5 & Jet stretched & 1.78 & 1.81 \\
PC-D5 & Steam drawn & 4.44 & 4.76 \\
PC-S2-D5 & Jet stretched and steam drawn & 18.44 & 15.65 \\
P & Freely spun & 0.81 & 0.53 \\
P-D5 & Steam drawn & 4.05 & 4.09 \\
P-S2-D5 & Jet stretched and steam drawn & 7.91 & 10.38 \\
\hline
\end{tabular}

I, diffraction intensity; A, area under the peak; eq, equatorial; mer, meridional.

orientation at polymer/CNT interface, which leads to higher crystal orientation compared to the structures without CNTs (Table 4). It confirms the possibility of crystal growth in the vicinity of CNTs.

\section{Mechanical Properties Young's Modulus}

The Young's modulus of PAN and PAN/CNT fibers increases with jet stretch (Table 5). Even with the lower crystallinity of jet-stretched PAN/CNT fibers in comparison with the corresponding PAN reference fibers (Table 3), Young's modulus of PC-S2 is still 9\% higher than that of P-S2, and Young's modulus of PC-S2.5 and P-S2.5 are almost identical. It seems that better orientation of the polymer chains and CNTs by application of jet stretch can result in the growth of crystals on CNTs and in increasing the crystal orientation along the fiber axis. Hence, the jetstretched PAN/CNT fibers have higher Young's modulus despite high porosity and low crystallinity compared to the corresponding PAN fibers.

When steam drawing is applied to coagulated fibers, Young's modulus has increased significantly due to improved polymer and crystal orientation as well as larger crystalline domain size and crystallinity even at high porosity (Table 5 and Figure 8A). Comparison of Young's modulus of the steam-drawn fibers at the ratio of 2.5 with the jet-stretched fibers at the ratio of 2.5 shows the larger impact of steam drawing on Young's modulus. It should be noted that steam drawing has larger impact on Young's modulus of PAN/CNT fibers than that of PAN reference fibers. For example, Young's modulus of PC-D2.5 fibers is $100 \%$ higher than that of PC-S2.5, whereas Young's modulus of P-D2.5 is $67 \%$ higher than that of P-S2.5. In addition, Young's modulus of PC-D2.5 is $20 \%$ higher than that of P-D2.5. It is evident that the presence of multiwalled CNTs inside the fiber structure has improved Young's modulus. The improvement in orientation of polymer chains and crystals in the fiber direction is the reason for the improvement of Young's modulus in PAN/CNT fibers.

\section{Tenacity}

The mechanical properties of fibers increase with application of elongational forces inside the coagulation bath through alignment of bonds of the chain backbone in the fiber direction and crystallinity enhancement. Jet stretch has increased the tenacity of PAN/CNT and PAN reference fibers up to 21 and $24 \%$, respectively (Table 5).

Steam drawing increases the tenacity of PAN/CNT and PAN fibers significantly (Table 5 and Figure 8B). For example, application of steam drawing at the ratio of 5 results in $320-350 \%$ increase in tenacity of PAN/CNT and PAN fibers. Steam drawing is more effective in improving the structure and mechanical properties than jet stretching. The tenacity of steam-drawn PAN/CNT composite fibers at the ratio of 2.5 is $65 \%$ higher than that of jet-stretched PAN/CNT fibers at the ratio of 2.5. Steam drawing at temperatures above the glass transition temperature of PAN has increased the freedom of movement of polymer chains and CNTs in PAN/CNT fibers. Crystalline domain size, crystallinity, and crystal orientation have also been improved as a result of better orientation of polymer chains in the fiber direction (Tables 3, 4). However, steam drawing has mostly increased the

TABLE 5 | Mechanical properties of PAN/CNT and PAN reference fibers at different stretching conditions.

\begin{tabular}{|c|c|c|c|c|c|c|c|}
\hline Condition & Sample name & Tenacity (cN/tex) & $\%$ CV & Strain at break $(\%)$ & $\% \mathrm{CV}$ & Young's modulus (cN/tex) & $\% \mathrm{Cv}$ \\
\hline Freely spun & PC & 2.4 & 11.6 & 40 & 16.2 & 50.1 & 16.4 \\
\hline Jet stretched & PC-S2.5 & 2.9 & 5.7 & 18 & 16.2 & 77.6 & 17.0 \\
\hline \multirow[t]{2}{*}{ Steam Drawn } & PC-D2.5 & 4.7 & 15.5 & 15 & 21.3 & 154.2 & 4.6 \\
\hline & PC-D5 & 9.9 & 6.9 & 28 & 6.3 & 156.2 & 16.6 \\
\hline \multicolumn{8}{|l|}{ PAN } \\
\hline Freely spun & $P$ & 2.4 & 8.9 & 33 & 14.0 & 48.1 & 11.6 \\
\hline \multirow[t]{2}{*}{ Jet stretched } & P-S2 & 2.7 & 7.5 & 36 & 7.0 & 67.2 & 7.0 \\
\hline & P-S2.5 & 3.2 & 2.8 & 34 & 7.2 & 78.1 & 8.2 \\
\hline \multirow[t]{2}{*}{ Steam drawn } & P-D2.5 & 6.8 & 5.9 & 25 & 13.2 & 130.2 & 15.0 \\
\hline & P-D5 & 10.8 & 7.0 & 35 & 6.9 & 153.8 & 16.4 \\
\hline
\end{tabular}




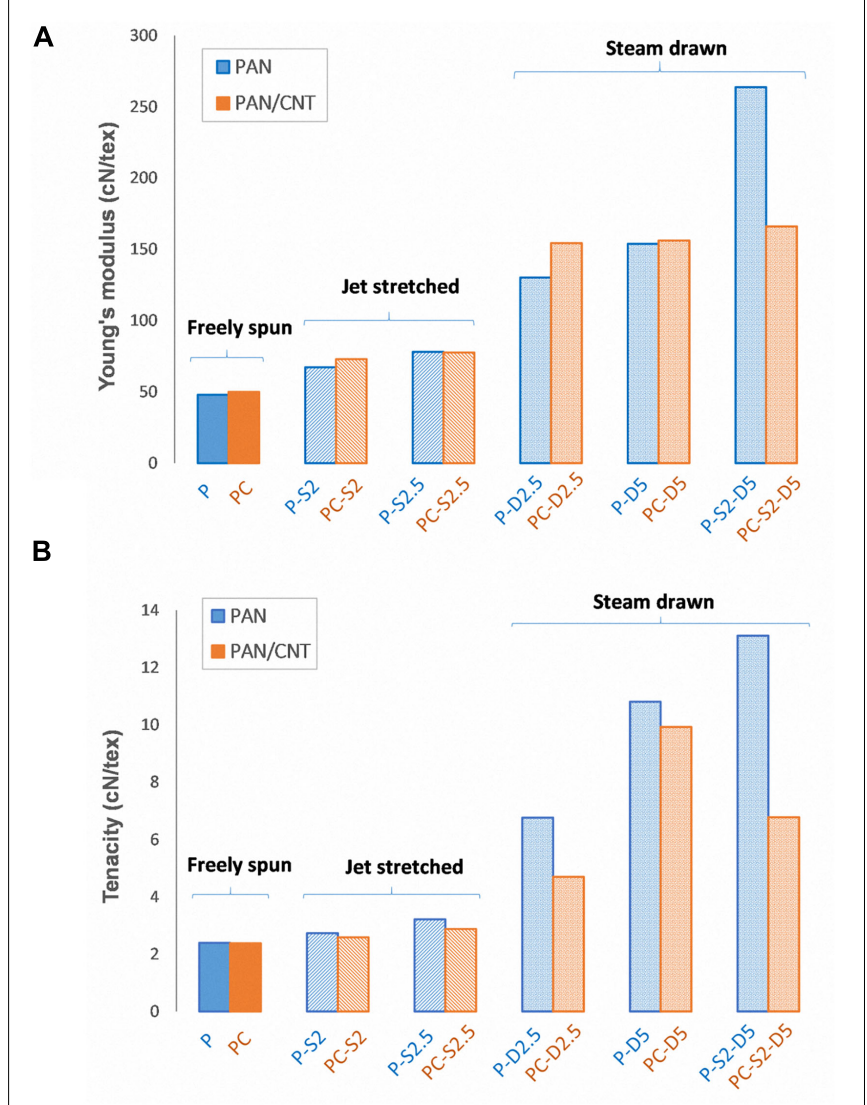

FIGURE 8 | Mechanical properties of PAN/CNT and PAN fibers at different stretching conditions, (A) Young's modulus, (B) Tenacity.

chain orientation of PAN reference fibers without increasing its crystallinity.

The highest tenacity of PAN/CNT fibers was achieved at steam drawing ratio of $5(9.928 \mathrm{cN} / \mathrm{tex})$, whereas PAN fibers had the highest tenacity when they were jet stretched at the ratio of 2 and then steam drawn at the ratio of 5 (13.112 cN/tex). Carbon nanotubes alter the optimum stretching conditions by affecting the orientation and crystallinity of structure. At similar steam drawing conditions, the tenacity of PAN fibers is higher than that of PAN/CNT fibers. Supposedly, CNTs have reduced the structure integrity, increasing the porosity. Not only does the polymer orientation affect the fiber strength, but also defects and voids in the molecular structure have an important role in mechanical properties of fibers (Ouyang et al., 2015). Macrovoids act as breaking points during stretching. To increase the tenacity of PAN/CNT fibers, it is necessary to control the coagulation and drying stage during the wet-spinning process to lower the porosity of the structure. Comparing the influence of various types of CNTs on the properties of PAN/CNT fibers, Chae et al. (2005) reported that multiwalled CNTs have a larger effect on tenacity and strain at break. However, the results of our work show that Young's modulus of fibers is more affected by the presence of multiwalled CNTs than tenacity.

When a combination of jet stretch at the ratio of 2 and steam drawing at the ratio of 5 is applied on fibers, the mechanical properties of PAN fibers exceeds that of PAN/CNT fibers. This can possibly be due to the negative effect of the high jet stretch ratio on porosity, morphology, crystalline structure, and mechanical properties of PAN/CNT fibers. Taking into account the outcomes of our previous paper (Mirbaha et al., 2019) as well as the observations in the current work, it is clear that for production of PAN/CNT fibers, lower jet-stretch ratios should be applied on fibers. In addition, controlling the porosity of structure by optimization of spinning conditions (i.e., coagulation, stretching and drying conditions) can lead to improvement in mechanical properties of PAN/CNT fibers compared to PAN fibers.

\section{CONCLUSION}

The present research on the impact of different stretching conditions on the crystalline structure and properties of wet-spun PAN/CNT microfibers led to the following outcomes:

(1) Steam drawing has a larger effect on crystalline structure and Young's modulus of PAN/CNT fibers than jet stretching, whereas jet stretching and steam drawing have a comparable effect on the crystalline structure of PAN fibers. Application of the steam draw ratio of 2.5 has improved Young's modulus of PAN/CNT fibers by a maximum of $100 \%$ in comparison to corresponding PAN/CNT fibers jet-stretched at the ratio of 2.5 .

(2) The effect of steam drawing on the crystalline structure and Young's modulus of PAN/CNT fibers is larger than its effect on PAN fibers. Young's modulus of PAN/CNT fibers could increase up to $19 \%$ higher than that of PAN fibers at a steam drawing ratio of 2.5. The existence of multiwalled CNTs inside the fibers has provided better crystalline structure of PAN/CNT fibers at lower steam drawing ratios compared to PAN fibers via nucleation of crystals around the CNTs. Improvement in the orientation of polymer chains and crystals in the fiber direction is the reason for the enhancement Young's modulus.

The results of this research can help to understand how different stretching stages in wet spinning (i.e., jet stretching inside coagulation bath and steam drawing after fiber coagulation) affect the structure and properties of composite PAN/CNT fibers in comparison to PAN fibers.

\section{DATA AVAILABILITY STATEMENT}

The raw data supporting the conclusions of this article will be made available by the authors, without undue reservation, to any qualified researcher. 


\section{AUTHOR CONTRIBUTIONS}

HM contributed to the conceptualization, doing the experiments, original draft preparation, writing, and editing the manuscript. PS contributed to editing the manuscript. MD'I contributed to the data acquiring. PS and MD'I contributed to the methodology. PS, MD'I, and HM contributed to the data analysis. PS, NP, and SA contributed to the providing resources. PN and NP contributed to the supervision and project administration. NP contributed to the original draft preparation and editing. All authors contributed to the article and approved the submitted version.

\section{REFERENCES}

Arbab, S., Noorpanah, P., Mohammadi, N., and Soleimani, M. (2008). Designing index of void structure and tensile properties in wet-spun polyacrylonitrile (PAN) Fiber. I. Effect of dope polymer or nonsolvent concentration. J. Appl. Polym. Sci. 109, 3461-3469. doi: 10.1002/app.28458

Arbab, S., Noorpanah, P., Mohammadi, N., and Zeinolebadi, A. (2011a). Exploring the effects of non-solvent concentration, jet-stretching and hot-drawing on microstructure formation of poly(acrylonitrile) fibers during wet-spinning. J. Polym. Res. 18, 1343-1351. doi: 10.1007/s10965-010-9537-7

Arbab, S., Noorpanah, P., Mohammadi, N., and Zeinolebadi, A. (2011b). Simultaneous effects of polymer concentration, jet-stretching, and hot-drawing on microstructural development of wet-spun poly(acrylonitrile) fibers. Polym. Bull. 66, 1267-1280. doi: 10.1007/s00289-010-0422-7

Bakhtiary Davijani, A. A., and Kumar, S. (2015). Ordered wrapping of poly(methyl methacrylate) on single wall carbon nanotubes. Polymer 70, 278-281. doi: 10.1016/j.polymer.2015.06.018

Basu-Dutt, S., Minus, M. L., Jain, R., Nepal, D., and Kumar, S. (2012). Chemistry of carbon nanotubes for everyone. J. Chem. Educ. 89, 221-229. doi: 10.1021/ ed1005163

Brown, T. R., Choudhury, R. P., Meree, C. E., Beckham, H. W., Kumar, S., and Shofner, M. L. (2014). Viscoelastic properties and structure of poly(acrylonitrile-co-methacrylic acid) polymer solutions for gel spinning at long aging times. J. Appl. Polym. Sci. 131:39821.

Cao, C., Chung, T., Chen, S., Dong, Z., and Jiang, S. (2004). The study of elongation and shear rates in spinning process and its effect on gas separation performance of poly(ether sulfone) (PES) hollow fiber membrane. Chem. Eng. Sci. 59:1053. doi: 10.1016/j.ces.2003.10.023

Chae, H. G., Sreekumar, T. V., Uchida, T., and Kumar, S. (2005). A comparison of reinforcement efficiency of various types of carbon nanotubes in polyacrylonitrile fiber. Polymer 46, 10925-10935. doi: 10.1016/j.polymer.2005. 08.092

Chen, J., Ge, H., Liu, H., Li, G., and Wang, C. (2010). The coagulation process of nascent fibers in PAN Wet spinning. J. Wuhan Uni. Technol. 25, 200-205. doi: 10.1007/s11595-010-2200-7

Dintcheva, N. T., Arrigo, R., Nasillo, G., Caponetti, E., and Mantia, F. P. L. (2011). On the role of extensional flow in morphology and property modifications of MWCNT/polyamide-based fibers. Macromol. Mater. Eng. 296, 645-657. doi: 10.1002/mame.201000355

Gissinger, J. R., Pramanik, C., Newcomb, B., Kumar, S., and Heinz, H. (2018). Nanoscale structure-property relationships of polyacrylonitrile/CNT composites as a function of polymer crystallinity and CNT diameter. ACS Appl. Mater. Interfaces 10, 1017-1027. doi: 10.1021/acsami.7b09739

Gong, Y., Du, R., Mo, G., Xing, X., Lu, C. X., and Wu, Z. (2014). In-situ Microstructural changes of polyacrylonitrile based fibers with stretching deformation. Polymer 55, 4270-4280. doi: 10.1016/j.polymer.2014. 06.073

Ji, B., Wang, C., and Wang, Y. (2007). Effect of jet stretch on polyacrylonitrile asspun fiber formation. J. Appl. Polym. Sci. 103, 3348-3352. doi: 10.1002/app. 25490

\section{FUNDING}

NP was supported by the European Commission under the FET Proactive ("Neurofibres") Grant No. 732344. NP and HM were also supported by the PRIN-2017 7TTP3S.

\section{ACKNOWLEDGMENTS}

We thank MCX beamline and the technical staff of the Italian synchrotron Elettra for collaboration.

Kang, D. W., and Ryu, S. H. (2013). Orientation of carbon nanotubes in polypropylene melt. Polym. Int. 62, 152-157. doi: 10.1002/pi.4270

Li, X., Qin, A., Zhao, X., Liu, D., Wang, H., and He, C. (2015). Drawing dependent structures, mechanical properties and cyclization behaviors of polyacrylonitrile and polyacrylonitrile/carbon nanotube composite fibers prepared by plasticized spinning. Phys. Chem. Chem. Phys. 17, 21856-21865. doi: 10.1039/c5cp02498f

Li, Y., Yu, Y., Liu, Y., and Lu, C. (2018). Interphase Development in Polyacrylonitrile/SWNT Nano-composite and its Effect on Cyclization and Carbonization for Tuning Carbon Structures. ACS Appl. Nano Mater. 1, 31053113. doi: 10.1021 /acsanm. 8 b00125

Lian, F., Liu, J., Ma, Z., and Liang, J. (2012). Stretching-induced deformation of polyacrylonitrile chains both in quasicrystals and in amorphous regions during the in situ thermal modification of fibers prior to oxidative stabilization. Carbon 50, 488-499. doi: 10.1016/j.carbon.2011.09.003

Lin, Y., Che, J., Pu, S., and Wang, K. (2019). Structural origins of mechanical strengthening in poly(phenylene sulfide)multiwalled carbon nanotube nanocomposites obtained via hot-stretching. Polym. Comp. 40, E589-E598.

Liu, H., Lv, F., Li, J., Cao, T., Wan, C., Zhang, W., et al. (2015). Two-stage drawing process to prepare high-strength and porous ultrahigh-molecularweight polyethylene fibers: cold drawing and hot drawing. J. Appl. Polym. Sci. 132:42823. doi: 10.1002/app.42823

Mai, F., Pan, D., Gao, X., Yao, M., Deng, H., Wang, K., et al. (2011). Extension-induced mechanical reinforcement in melt-spun fibers of polyamide 66/multiwalled carbon nanotube composites. Polym. Int. 60, 1646-1654. doi: 10.1002/pi.3144

Mikolajczyk, T., Szparaga, G., Bogun, M., Fraczek-Szczypta, A., and Blazewicz, S. (2010). Effect of spinning conditions on the mechanical properties of polyacrylonitrile fibers modified with carbon nanotubes. J. Appl. Polym. Sci. 115, 3628-3635. doi: 10.1002/app.31414

Minus, M. L., Chae, H. G., and Kumar, S. (2012). Polyethylene crystallization nucleated by carbon nanotubes under shear. ACS Appl. Mater. Interface 4, 326-330. doi: 10.1021/am2013757

Mirbaha, H., Nourpanah, P., Scardi, P., D’incau, M., Greco, G., Valentini, L., et al. (2019). The impact of shear and elongational forces on structural formation of polyacrylonitrile/carbon nanotubes composite fibers during wet spinning process. Materials 12:2797. doi: 10.3390/ma12172797

Ouyang, Q., Chen, Y., Wang, X., Ma, H., Li, D., and Yang, J. (2015). Supramolecular structure of highly oriented wet-spun polyacrylonitrile fibers used in the preparation of high-performance carbon fibers. J. Polym. Res. 22:229.

Ouyang, Q., Chen, Y. S., Zhang, N., Mo, G. M., Li, D. H., and Yan, Q. (2011). Effect of jet swell and jet stretch on the structure of wet-spun polyacrylonitrile fiber. J. Macromol. Sci. B Phys. 50, 2417-2427. doi: 10.1080/00222348.2011.564104

Rebuffi, L., Plaisier, J. R., Abdellatief, M., Lausi, A., and Scardi, P. (2014). MCX: a synchrotron radiation beamline form X-ray diffraction line profile analysis. Z. Anorg. Allg. Chem. 640, 3100-3106. doi: 10.1002/zaac.201400163

Sahin, K., Fasanella, N. A., Chasiotis, I., Lyons, K. M., Newcomb, B. A., Kamath, M. G., et al. (2014). High strength micron size carbon fibers from polyacrylonitrile-carbon nanotube precursors. Carbon 77, 442-453. doi: 10. 1016/j.carbon.2014.05.049 
Sapalidis, A., Sideratou, Z., Panagiotaki, K. N., Sakellis, E., Kouvelos, E. P., Papageorgiou, S., et al. (2018). Fabrication of antibacterial poly(Vinyl Alcohol) nanocomposite films containing dendritic polymer functionalized multi-walled carbon nanotubes. Front. Mater. 5:11. doi: 10.3389/fmats.2018. 00011

Ward, I. M. (1997). Structure and Properties of Oriented Polymers. Netherlands: Springer.

Wei, H., Suo, X., Lu, C., and Liu, Y. (2020). A comparison of coagulation and gelation on the structures and stabilization behaviors of polyacrylonitrile fibers. J. Appl. Polym. Sci. 137:48671. doi: 10.1002/app.48671

Wilms, C., Seide, G., and Gries, T. (2013). The relationship between process technology, structure development and fibre properties in modern carbon fibre production. Chem. Eng. Trans. 32, 1609-1614.

Zeng, X., Hu, J., Zhao, J., Zhang, Y., and Pan, D. (2007). Investigating the jet stretch in the wet spinning of PAN fiber. J. Appl. Polym. Sci. 106, 2267-2273. doi: 10.1002/app.26929
Zhang, Y., Song, K., Meng, J., and Minus, M. L. (2013). Tailoring polyacrylonitrile interfacial morphological structure by crystallization in the presence of singlewall carbon nanotubes. ACS Appl. Mater. Interface 5, 807-814. doi: 10.1021/ am $302382 \mathrm{~m}$

Conflict of Interest: The authors declare that the research was conducted in the absence of any commercial or financial relationships that could be construed as a potential conflict of interest.

Copyright (c) 2020 Mirbaha, Scardi, D'Incau, Arbab, Nourpanah and Pugno. This is an open-access article distributed under the terms of the Creative Commons Attribution License (CC BY). The use, distribution or reproduction in other forums is permitted, provided the original author(s) and the copyright owner(s) are credited and that the original publication in this journal is cited, in accordance with accepted academic practice. No use, distribution or reproduction is permitted which does not comply with these terms. 